\title{
ESSENCE-Q - used as a screening tool for neurodevelopmental problems in public health checkups for young children in south Japan
}

This article was published in the following Dove Press journal:

Neuropsychiatric Disease and Treatment

10 May 2017

Number of times this article has been viewed

\author{
Yuhei Hatakenaka ${ }^{1,2}$ \\ Hitoshi Ninomiya ${ }^{3}$ \\ Eva Billstedt ${ }^{2}$ \\ Elisabeth Fernell ${ }^{2}$ \\ Christopher Gillberg ${ }^{2}$ \\ 'Kochi Gillberg Neuropsychiatry \\ Centre, Kochi Prefectural Medical and \\ Welfare Center, Kochi, Japan; ${ }^{2}$ Gillberg \\ Neuropsychiatry Centre, Sahlgrenska \\ Academy, University of Gothenburg, \\ Gothenburg, Sweden; Integrated \\ Centre for Advanced Medical \\ Technologies, Kochi University \\ Medical School, Kochi, Japan
}

Background: Screening for developmental disorders is an important task for Child Health Care. The concept of ESSENCE (early symptomatic syndromes eliciting neurodevelopmental clinical examinations) was created to cover all types of early developmental disorders and the ESSENCE-Questionnaire (ESSENCE-Q containing 12 questions with possible total scores ranging from 0 to 22) was developed as a tool for early detection of these disorders. The aim of this study was to perform a validation study in a public health situation in Japan.

Methods: The psychometric properties of the ESSENCE-Q, completed by mothers, public health nurses (PHNs), and psychologists at 18-month ( $\mathrm{n}=143$ children) and 36-month ( $\mathrm{n}=149$ children) checkups were evaluated in a small city of Japan. Results were validated against clinical ESSENCE diagnoses. Receiver operating characteristic curves were generated and compared by using the area under the curve (AUC). Optimal cutoff values were explored.

Results: At the 18 -month checkup, AUC by mothers was 0.72 , by PHNs 0.86 , and by psychologists 0.82 . An optimal cutoff was 3 with a high negative predictive value (NPV). At the 36-month checkup, AUC by mothers was 0.57 , by PHNs 0.82 , and by psychologists 0.87 . Optimal cutoff was 2 with high NPV.

Conclusion: The ESSENCE-Q completed by PHNs and psychologists had good diagnostic validity. The results suggested that almost all children scoring under cutoff would not have any ESSENCE problems/diagnoses.

Keywords: ESSENCE, questionnaire, public health nurse, receiver operating characteristic, ROC, area under the curve, AUC, optimal cutoff, negative predictive value, NPV

\section{Introduction}

Screening for chronic diseases has been defined as:

[...] the presumptive identification of unrecognized disease or defect by application of tests, examinations or other procedures, which can be applied rapidly [...]. Persons with positive or suspicious finding must be referred to their physician for diagnosis and necessary treatment. ${ }^{1}$

If "disease or defect" is changed to "neurodevelopmental disorder or problem" and "to their physician" to "for neurodevelopmental assessment," then this definition can also be applied to developmental screening. The main aim of developmental screening was to assess a child's risk of developmental disorders. ${ }^{2,3}$ Early identification of neurodevelopmental problems is one of the most important tasks for public health. ${ }^{4}$ Many outcome studies of children with developmental disorders have demonstrated that early tailored interventions are important to maximize the
Correspondence: Yuhei Hatakenaka Department of Human Sciences, Faculty of Law and Letter, University of the Ryukyus, I Senbaru, Nishihara-cho, Okinawa, 903-0213, Japan

Tel +8I 988958257

Email yuhei.hatakenaka@gmail.com $\mathrm{BY}$
hereby accept the Terms. Non-commercial uses of the work are permitted without any further permission from Dove Medical Press Limited, provided the work is properly attributed. For permission for commercial use of this work, please see paragraphs 4.2 and 5 of our Terms (https://www.dovepress.com/terms.php). 
individual child's potential. ${ }^{3}$ There is growing evidence that, even when a precise diagnosis cannot be established, early intervention taking the child's basic impairment into account is important. ${ }^{5-8}$

ESSENCE (early symptomatic syndromes eliciting neurodevelopmental clinical examinations) is a concept that was introduced with a view to alert clinicians and researchers to the complexity and overlap of different neurodevelopmental disorders/problems including autism spectrum disorder (ASD), attention-deficit/hyperactivity disorder (ADHD), oppositional defiant disorder (ODD), tic disorders (TDs), developmental coordination disorder (DCD), speech and language disorder (SLD), intellectual developmental disorder (IDD), borderline intellectual functioning (BIF), and other non-specific learning difficulties (LDs). ${ }^{9}$ If a child before 5 years of age has at least one (usually several) problem lasting more than several months in the following 12 areas - general development, communication/language, social interrelatedness, perception, motor coordination, attention/“listening," activity, "behavior," mood, feeding, sleep, and/or episodes/absences - this should be seen as a red flag for possible neurodevelopmental disorders. Unrecognized and "untreated" ESSENCE may predispose to adult psychiatric and/or physical disorders. With regard to prevalence, at least $10 \%$ of children aged $<18$ years are affected by ESSENCE. ${ }^{9}$ Identifying problems under the ESSENCE concept in young children and monitoring child development would possibly reduce the risk of later maladjustment.

The ESSENCE-Questionnaire (ESSENCE-Q) is a screening tool designed to help clinicians and researchers to identify children with ESSENCE (or neurodevelopmental) problems. ${ }^{10}$ The ESSENCE-Q is intended for use in both clinical practice and population research. It is proposed to be useful as a questionnaire to be completed by parents and/or caregivers or as a brief interview by a specialist. It has already been shown that the ESSENCE-Q can be useful as a parent questionnaire in a neurodevelopmental clinic. ${ }^{11}$ It could also be useful in a public health setting as a parent questionnaire or as an interview questionnaire and/or as the basis for an observation record.

Screening at child health care centers has proven to be a valuable method to identify developmental disorders in young children. Previous studies ${ }^{12}$ have demonstrated the use of a language screening, performed by the health nurse, and that such screening identifies also children with other ESSENCE problems. Such screening methods include the professional assessment supplemented by reports from parents. The value of the ESSENCE-Q lies in its short format and its broad approach, covering different developmental areas. It can be used to obtain both parents' and professionals' concerns at the child's health checkups and will thereby increase the validity of the screening.

In Japan, according to the Maternal and Child Health Law, ${ }^{13}$ all children are invited to take part in health checkups, first during 18-24 months ("18-month checkups") and second during 36-48 months ("36-month checkups"). Municipalities have responsibility for these checkups, and the rate of attendance is $\sim 95 \% .{ }^{14}$ Therefore, these checkups provide good opportunities to identify the children with neurodevelopmental disorders. ${ }^{15}$ The ESSENCE-Q in 18-month and 36-month checkups in a small city of Japan was used as a parent questionnaire to mothers and as a parent-interview and child-observation tool used by public health nurses (PHNs) and psychologists who were specialized in neurodevelopmental disorders (specialized psychologists).

The aims of the present study were to 1) evaluate the validity of the ESSENENCE-Q completed by mothers, PHNs, and specialized psychologists in a public health setting and 2) explore an optimal cutoff score of the ESSENCE-Q at 18- and 36-month checkups.

\section{Methods}

This is a prospective, population-based, observational cohort study. Data on two separate groups of children who came to health care centers in Kami City, Kochi, Japan, from April 2014 to March 2015 were collected. In Kami City, parents of children who were 18 or 42 months of age during the period were invited to 18 - or 36-month checkups. PHNs in the city have been working with specialized psychologists, and they have had on-the-job training in screening of neurodevelopmental disorders for several years. They also participated in seminars about ESSENCE and related problems, including about the ESSENCE-Q.

\section{ESSENCE-Q}

\section{Procedures}

The ESSENCE-Q is a brief one-page "quick and easy" questionnaire containing 12 items covering concerns relating to the above-mentioned 12 areas. Response options of "Yes," "Maybe/A little," or "No" should be checked for each item. It is not a diagnostic instrument or an instrument that can be used as a proxy for diagnoses, but a screening tool to be used in order not to overlook children who need further assessments by a developmental specialist. ${ }^{10}$

All ESSENCE-Q data from mothers who came with their child to the 18-month or the 36-month checkup were collected. 
PHNs and specialized psychologists were engaged in these checkups. The mothers, PHNs, and the specialized psychologists scored the child's ESSENCE-Q blind to each other.

The ESSENCE-Q was sent to mothers before the checkup, and they were asked through letter to complete the questionnaire. These ESSENCE-Qs (ESSENCE-Q-M) were gathered by receptionists at the checkups. Then, at the checkups, the PHNs completed the ESSENCE-Q (ESSENCEQ-N) through interview with the mothers and through direct observation of the child without any knowledge about the ESSENCE-Q-M. Third, specialized psychologists completed the ESSENCE-Q (ESSENCE-Q-P) through interview with the mothers and through direct observation of the child without any knowledge about the ESSENCE-Q-M and the ESSENCE-Q-N.

The PHNs and the specialized psychologists used the ESSENCE-Q statements as a template for their interviews, and sometimes added simple questions (eg, "When did your child start walking?"). After the interview and the observation, they noted their concerns in the ESSENCE-Q.

\section{ESSENCE diagnoses}

At the end of the checkups, a pediatrician with training in the field of ESSENCE examined the child and interviewed the mother. The child's entire records with regard to development from birth were checked as were the three ESSENCE-Qs. Taking all this information into consideration, the pediatrician made a decision as to whether or not the child needed to go for further neurodevelopmental examinations. Children targeted for further examinations were then examined - on another day at the same health center - by a specialized child neuropsychiatrist (including interview with the mother). If there was any remaining concern regarding neurodevelopmental disorders, the child was referred to a neurodevelopmental clinic for further investigation by the psychiatrist and a team including occupational therapists and psychologists. The time periods between the secondary checkup and the first assessment at the neurodevelopmental clinic were $<4$ months (in most cases, 2 months). The child neuropsychiatrist was not blind to the results of the ESSENCE-Qs, but these results did not form part of his referral decision.

At the neurodevelopmental clinic, assessments covered all developmental areas included under the ESSENCE umbrella. Overall development, motor and perceptual performance, social communication and related behaviors, social interest, joint attention, imitation, play, reciprocal affective behavior, and insistence on sameness/stereotypies ${ }^{16-18}$ were assessed for all children at the first two to three visits.
For cognitive assessment, the Kyoto Scale of Psychological Development 2001 (KSPD2001) ${ }^{19}$ was used. KSPD is an individualized face-to-face test to assess a child's development in the areas of fine and gross motor functions, non-verbal reasoning, visuospatial perceptions, interpersonal relationships, socializations, and verbal abilities. ${ }^{19}$ KSPD2001 has excellent psychometric properties, and the results are closely correlated with the Bayley Scales of Infant Development. ${ }^{20}$ For the assessment of social and communication development, the Diagnostic Interview for Social and Communication Disorders $(\mathrm{DISCO})^{21}$ was used. The DISCO is widely used as an assessment tool for autism spectrum conditions. The interview is semistructured and covers a wide range of behaviors associated with the ASD phenotype. It is suitable for use with all ages and levels of ability. It enables to identify specific features found in ASD. The Strengths and Difficulties Questionnaire (SDQ) ${ }^{22}$ for parents of 2- to 4-year olds was used. SDQ covers child mental health and developmental aspects, including child behavior, emotions, and relationships. It also addresses impact and duration of symptoms, distress in the child, impairment in different settings, and burden to others. SDQ can be used as a screening tool for several types of neurodevelopmental disorders, such as ASD and ADHD. Unstructured clinical observations at the clinic and reports/interviews from parents and preschool teachers were collected throughout the examination period. Motor-perceptual performance was examined at clinical observations. The diagnostic evaluations were done at least at five different sessions separated by a minimum of 2 weeks. ${ }^{23}$ For the children who were referred from the 18-month checkups, definite diagnoses were given at or after 30 months. The International Statistical Classification of Diseases-10/Diagnostic and Statistical Manual of Mental Disorders-IV criteria for diagnoses of child psychiatric/ neurodevelopmental disorders were used throughout.

\section{Statistical analysis}

ESSENCE-Q items were rated as 0 for "No," 1 for "Maybe/a little," and 2 for "Yes." The range of possible scores was $0-24$. If four or more $(>10 \%)$ of the 36 items, collapsed from the three ESSENCE-Qs, were unchecked by mother, PHS, or specialized psychologist, then this case was excluded. Unchecked items were otherwise rated as 0 . These overall ESSENCE-Q scores were used as continuous variables and generated receiver operating characteristic (ROC) curves and compared the area under the curve (AUC) to evaluate the validity of ESSENCE-Q-M, ESSENCE-Q-N, and ESSENCE-Q-P separately. In addition, the optimal cutoff values for screening in health checkups from the ROC 
curves were explored. For a developmental screening, the best sensitivity and specificity balance is around $0.70-0.80$ for both. ${ }^{5,24}$ Sensitivity should be higher than specificity so as not to miss children with problems. ${ }^{25}$ When an optimal cutoff value fulfilling these conditions was found, the sensitivity, specificity, positive predictive value (PPV), and negative predictive value (NPV) were calculated. The data from 18- and 36-month checkups were analyzed separately. All statistical analyses were done by $\mathrm{R}$ version 3.1.3 (The R Foundation for Statistical Computing, Vienna, Austria). Sensitivity, specificity, PPV, and NPV were expressed with a $95 \%$ confidence interval $(\mathrm{CI})$.

\section{Ethics}

The institutional ethical boards of Kochi Prefectural Medical and Welfare Centre approved the study with parents' written informed consent, No 25-2056.

\section{Results}

\section{Characteristics of study population}

I8-month checkups (Figure I)

During the study period, all 152 children ( 79 boys and 73 girls) were invited to the 18-month checkup and 143 children (75 boys and 68 girls, mean age 18.7, standard deviation [SD] 0.9) participated. The attendance rate was $94 \%$. Of the 143 children, 50 (27 boys and 23 girls) (35\%) were invited to come to the secondary checkup due to developmental concerns. However, eight (three boys and five girls) did not come to the secondary checkup. Of the remaining 42 children, 21 (10 boys and 11 girls) were regarded to have normal development and 21 (14 boys and 7 girls) were referred to the neurodevelopmental clinic. One boy's family moved out from the city and, as a result, 20 (13 boys and 7 girls) came to the clinic.

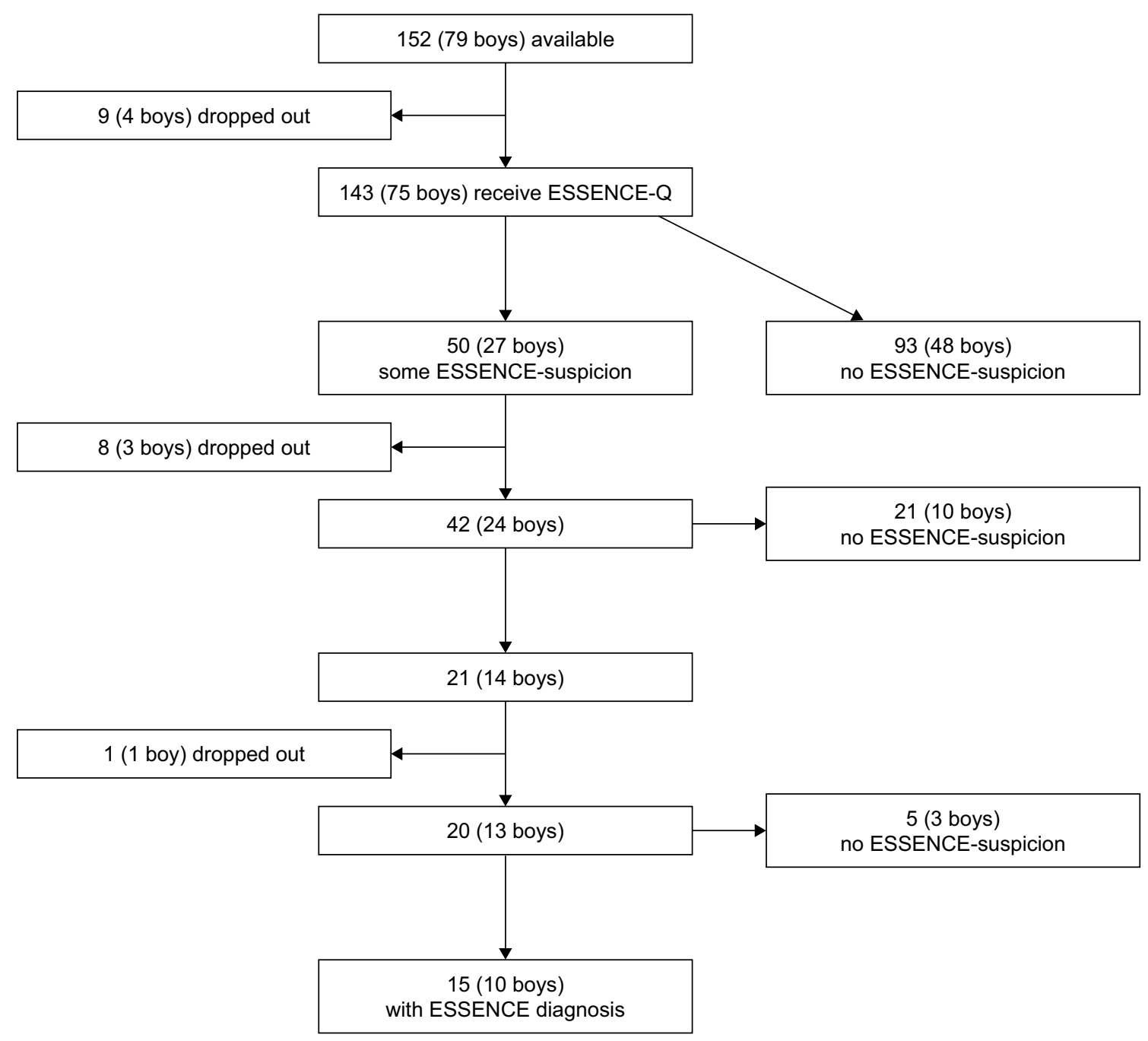

Figure I Flowchart of the children in I8-month checkups.

Abbreviation: ESSENCE, early symptomatic syndromes eliciting neurodevelopmental clinical examinations; ESSENCE-Q, ESSENCE-Questionnaire. 
Neurodevelopmental diagnoses in the children of the I8-month checkups

Of the 20 children who came to the clinic, five (three boys and two girls) were considered to have a normal developmental trajectory and 15 children ( 10 boys and 5 girls) were given neurodevelopmental diagnoses $(10 \%$ of all participants). One third had intellectual problems (IDD, BIF) and one third had ADHD. Two had ASD. Table 1 shows diagnostic and other information of the 20 children who came to the clinic.

Three of the 143 children (three boys without a diagnosis) were excluded because of missing data. The eight children who did not come for the second assessment and the child who moved from the area were excluded (because it could not be ascertained whether or not they actually had an ESSENCE diagnosis), leaving 131 (63 boys and 68 girls, mean age 18.7 , SD $0.8,92 \%$ of all the participants) for statistical analyses.

\section{"Thirty six-month checkups" performed at a mean age of 42 months (Figure 2)}

During the period, 158 children ( 77 boys and 81 girls) were invited to the "36-month checkups" (which in reality occurred at 42 months in the majority of cases) and 149 children ( 73 boys and 76 girls, mean age 42.0 months,
SD 1.0) participated. The attendance rate was $94 \%$. Of the 149 children, 38 ( 19 boys and 19 girls) (26\%) were invited to come to the secondary checkup due to developmental concerns. However, five (five boys) did not come to the secondary checkup. In the remaining 33 children, 17 were regarded to have a normal development ( 6 boys and 11 girls) and 16 ( 8 boys and 8 girls) were referred to the neurodevelopmental clinic. A family of a boy and another family of a girl moved out from the area and, as a result, 14 ( 7 boys and 7 girls) came to the clinic.

\section{Neurodevelopmental diagnoses in the children of the 42-month checkups}

All children who came to the clinic were given neurodevelopmental diagnoses ( $9 \%$ of all participants). Twelve had ADHD and two had ASD. Ten had two or more diagnoses. Table 2 shows diagnostic and other information of the 14 children who were referred to the clinic.

Three of the 149 children (three boys, one of whom had a diagnosis) were excluded because of missing data. Five children who did not come to the secondary assessment and two children who did not come for full clinical assessment were also excluded, leaving 139 (64 boys and 75 girls, mean age 42.0 , SD 1.0, 93\% of all the participants) for statistical analyses.

Table I Diagnosis and background information of the 21 children referred to the clinic from I8-month checkups

\begin{tabular}{|c|c|c|c|c|}
\hline Number & Gender & $\begin{array}{l}\text { Age at the first } \\
\text { checkup (months) }\end{array}$ & $\begin{array}{l}\text { Age at the first } \\
\text { visit (months) }\end{array}$ & $\begin{array}{l}\text { ESSENCE diagnoses/conditions } \\
\text { at age (months) }\end{array}$ \\
\hline $\mathrm{I}$ & Boy & 19 & 21 & Normal developmental trajectory (44) \\
\hline 2 & Boy & 18 & 21 & Normal developmental trajectory (33) \\
\hline 3 & Boy & 18 & 19 & ASD (33) \\
\hline 4 & Boy & 18 & 26 & ADHD (4I), IDD (4I) \\
\hline 5 & Boy & 18 & 20 & ADHD (39), DCD (39) \\
\hline 6 & Boy & 19 & 38 & BIF (39) \\
\hline 7 & Boy & 19 & 20 & ASD (36) \\
\hline 8 & Boy & 18 & 21 & Normal developmental trajectory (30) \\
\hline 9 & Boy & 21 & 22 & IDD (30) \\
\hline 10 & Boy & 19 & 22 & IDD (33) \\
\hline 11 & Boy & 19 & 25 & ADHD (32) \\
\hline 12 & Boy & 18 & 29 & SLD (30) \\
\hline 13 & Boy & 18 & 20 & IDD (30) \\
\hline $14^{*}$ & Boy & 18 & N/A & $\mathrm{N} / \mathrm{A}$ \\
\hline 15 & Girl & 18 & 21 & ADHD (42), SLD (43) \\
\hline 16 & Girl & 19 & 23 & Normal developmental trajectory (38) \\
\hline 17 & Girl & 18 & 22 & SLD (37) \\
\hline 18 & Girl & 19 & 28 & Normal developmental trajectory (30) \\
\hline 19 & Girl & 18 & 20 & SLD (42) \\
\hline 20 & Girl & 18 & 21 & ADHD (36) \\
\hline 21 & Girl & 18 & 19 & IDD (30) \\
\hline
\end{tabular}

Note: *Lost to follow-up due to changing address.

Abbreviations: ADHD, attention-deficit/hyperactivity disorder; ASD, autism spectrum disorder; BIF, borderline intellectual functioning; DCD, developmental coordination disorder; ESSENCE, early symptomatic syndromes eliciting neurodevelopmental clinical examinations; IDD, intellectual developmental disorder; SLD, speech and language disorder; N/A, not applicable. 


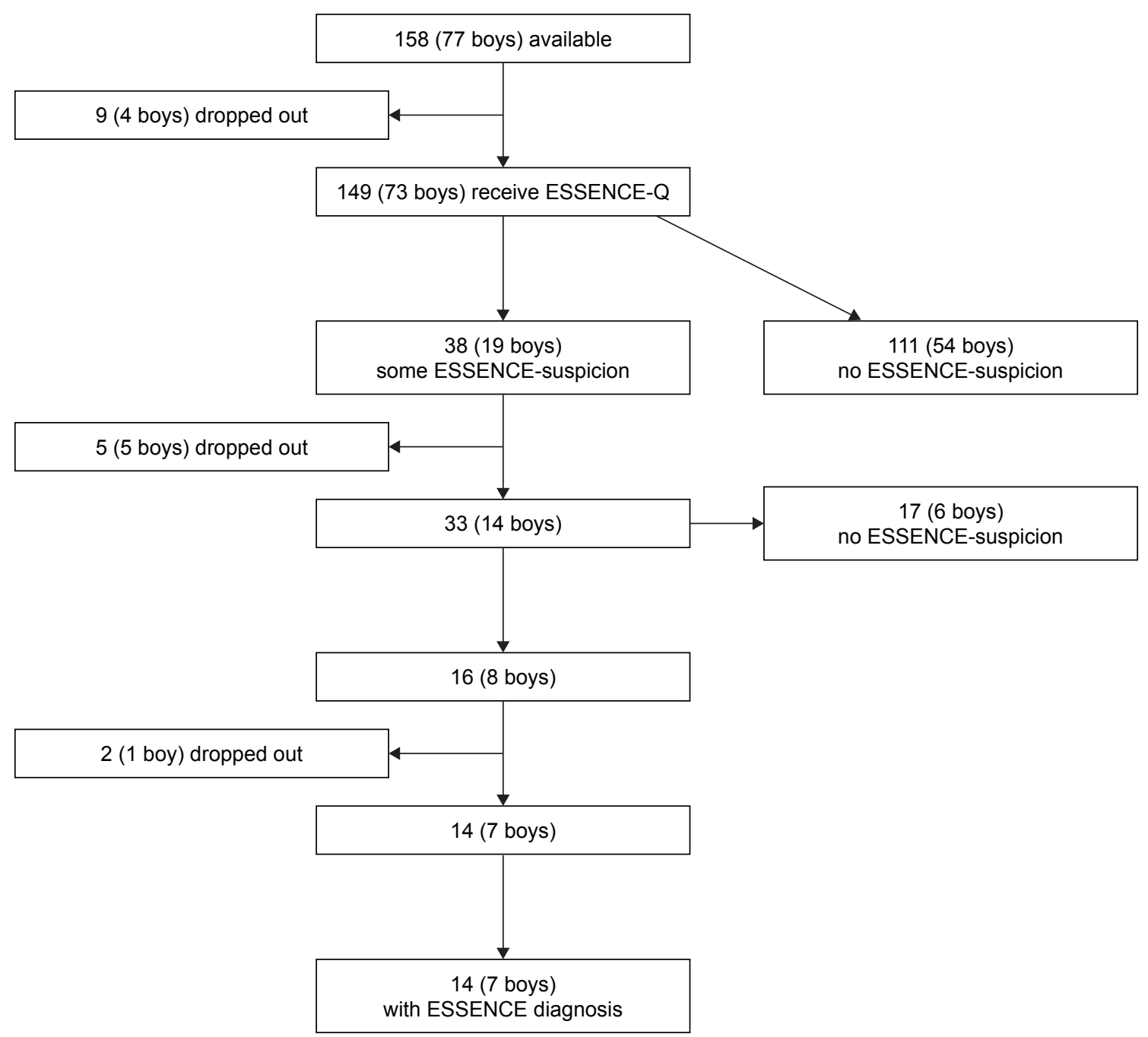

Figure 2 Flowchart of the children in 36-month checkups.

Abbreviation: ESSENCE, early symptomatic syndromes eliciting neurodevelopmental clinical examinations; ESSENCE-Q, ESSENCE-Questionnaire.

\section{ESSENCE-Q scores}

\section{I8-month checkups (Figure 3, Table 3)}

Overall ESSENCE-Q-M, ESSENCE-Q-N, and ESSENCE-Q-P scores produced AUC values $(95 \% \mathrm{CI})$ of $0.69(0.52-0.86), 0.92$ (0.86-0.97), and 0.85 (0.74-0.96), respectively. For ESSENCE$\mathrm{Q}-\mathrm{M}$, an optimal cutoff value that fulfilled the conditions mentioned above was not found. For ESSENCE-Q-N, the optimal cutoff value was set at 3, which showed a sensitivity of 0.93 (95\% CI: $0.66-1.00)$ a specificity of $0.74(95 \%$ CI: 0.66-0.82), a PPV 0.30 (95\% CI: 0.17-0.46), and an NPV 0.99 (0.94-1.00). For ESSENCE-Q-P, an optimal cutoff value was set as 3, which showed a sensitivity of 0.86 (95\% CI: 0.57-0.98), a specificity of 0.75 (95\% CI: $0.66-0.83)$, a PPV of 0.29 (95\% CI: 0.16-0.46), and an NPV of 0.98 (95\% CI: 0.92-1.00).

\section{6-month checkups (Figure 4, Table 4)}

Overall ESSENCE-Q-M, ESSENCE-Q-N, and ESSENCEQ-P scores produced AUC values $(95 \% \mathrm{CI})$ of 0.63
(0.46-0.81), $0.84(0.73-0.95)$, and $0.82(0.69-0.94)$. For ESSENCE-Q-M, an optimal cutoff value that fulfilled the conditions mentioned above was not found. For ESSENCEQ-N, the optimal cutoff value was set as 2, which showed a sensitivity of 0.86 (95\% CI: $0.57-0.98)$, a specificity 0.70 (95\% CI: $0.61-0.76)$, a PPV of 0.24 (95\% CI: $0.13-0.38$ ), and an NPV of 0.98 (95\% CI: 0.92-1.00). For ESSENCEQ-P, the optimal cutoff value was set as 2 , which showed a sensitivity of 0.86 (95\% CI: $0.57-0.98)$, a specificity of 0.66 (95\% CI: $0.57-0.75)$, a PPV of 0.22 (95\% CI: $0.12-0.36$ ), and an NPV of 0.98 (95\% CI: 0.92-1.00).

\section{Differences between boys and girls}

Results were subdivided according to gender. There was a clear trend toward boys having "better" validity data than the girls. In the 18-month checkups, sensitivity was 0.89 and 0.89 for boys compared to 1.00 and 0.80 for girls at ESSENCE-Q-N and ESSENCE-Q-P, respectively. 
Table 2 Diagnosis and background information of the 16 children referred to the clinic from 36-month checkups

\begin{tabular}{lllll}
\hline No & Gender & $\begin{array}{l}\text { Age at the first } \\
\text { checkup (months) }\end{array}$ & $\begin{array}{l}\text { Age at the first } \\
\text { visit (months) }\end{array}$ & $\begin{array}{l}\text { ESSENCE diagnoses/ } \\
\text { conditions at age (months) }\end{array}$ \\
\hline 1 & Boy & 41 & 46 & ASD (48), DCD (48), Tics (48) \\
2 & Boy & 42 & 47 & BIF (49), ADHD (56) \\
3 & Boy & 42 & 43 & ASD (45), ADHD (58) \\
4 & Boy & 42 & 43 & DCD (44), ADHD (5I) \\
5 & Boy & 41 & 45 & ADHD (5I), SLD (5I) \\
6 & Boy & 41 & 44 & ADHD (57), SLD (57) \\
$7 *$ & Boy & 43 & N/A & N/A \\
$8 * *$ & Boy & 42 & 44 & ADHD (52), BIF (52) \\
9 & Girl & 43 & 51 & ADHD (59) \\
10 & Girl & 43 & 45 & DCD (45), Tics (45), SAD (45), \\
& Girl & & 46 & Congenital tremor (45) \\
11 & Girl & 41 & 44 & ADHD (56) \\
12 & Girl & 43 & 45 & ADHD (59), BIF (59) \\
13 & Girl & 42 & 45 & ADHD (59) \\
14 & Girl & 42 & N/A & ADHD (59) \\
$15 *$ & Girl & 43 & 47 & N/A \\
\hline 16 & 41 & ADHD (53), RAD (53) &
\end{tabular}

Notes: *Lost to follow-up due to changing address. **Excluded from ROC analysis because of missing data.

Abbreviations: ADHD, attention-deficit/hyperactivity disorder; ASD, autism spectrum disorder; BIF, borderline intellectual functioning; DCD, developmental coordination disorder; ESSENCE, early symptomatic syndromes eliciting neurodevelopmental clinical examinations; N/A, not applicable; RAD, reactive attachment disorder; SAD, social anxiety disorder; SLD, speech and language disorder; ROC, receiver operating characteristic.

Specificity was 0.75 and 0.71 for boys compared to 0.74 and 0.79 for girls. PPV was 0.35 and 0.32 for boys compared to 0.25 and 0.25 for girls. NPV was 0.98 and 0.98 for boys compared to 1.00 and 0.98 for girls. In the 36-month checkups, sensitivity was 1.00 and 0.86 for boys compared to 0.71 and 0.86 for girls at ESSENCE-Q-N and ESSENCEQ-P, respectively. Specificity was 0.75 and 0.70 for boys

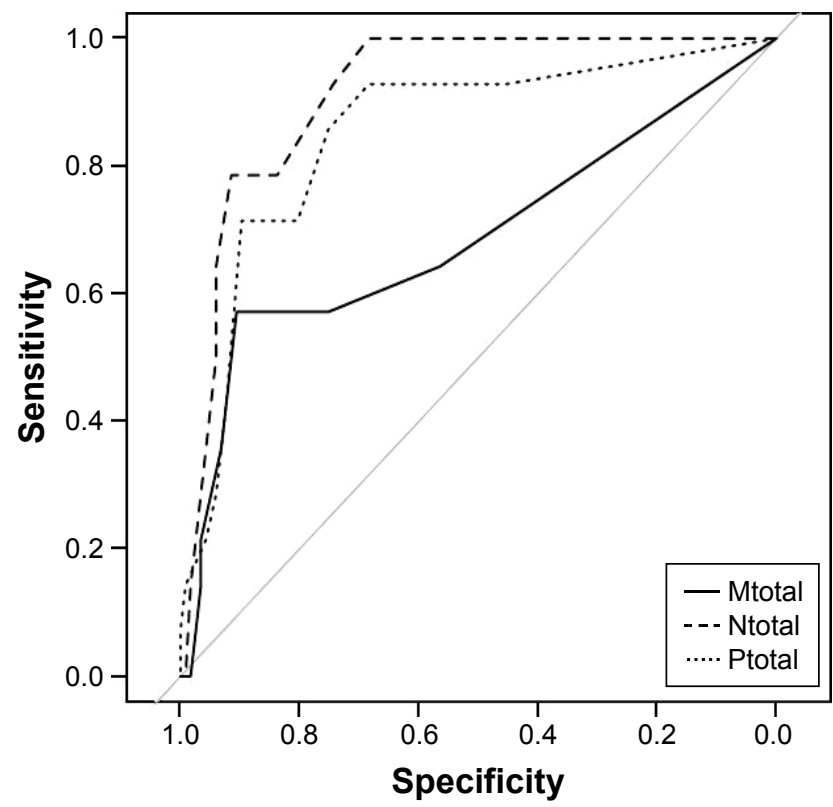

Figure 3 ROC curves for predicting ESSENCE diagnoses at 18-month checkups based on overall ESSENCE-Q score in ESSENCE-Q-M (Mtotal), ESSENCE-Q-N (Ntotal), and ESSENCE-Q-P (Ptotal).

Abbreviations: ESSENCE, early symptomatic syndromes eliciting neurodevelopmental clinical examinations; ESSENCE-Q, ESSENCE-Questionnaire; ROC, receiver operating characteristic. compared to 0.65 and 0.63 for girls. PPV was 0.33 and 0.26 for boys compared to 0.17 and 0.19 for girls. NPV was 1.00 and 0.98 for boys compared to 0.96 and 0.98 for girls. The significance of the differences was not tested because of small numbers.

\section{Attrition results}

Of the nine children in the 18-month checkups and the seven children in the 36-month checkups who could not be diagnostically assessed (and who were suspected to be cases), five and six respectively were screened positive. This rate ( $69 \%$ of all who dropped out after initial screening) is higher than the rate $(36 \%)$ for those who were retained for the statistical analyses.

Table 3 AUC, sensitivity, specificity, PPV, and NPV of the three ESSENCE-Qs in 18-month checkups

\begin{tabular}{|c|c|c|c|}
\hline $\begin{array}{l}18 \text { months } \\
(n=131)\end{array}$ & ESSENCE-Q-M & ESSENCE-Q-N & ESSENCE-Q-P \\
\hline AUC & $0.69(0.52-0.86)$ & $0.92(0.86-0.97)$ & $0.85(0.74-0.96)$ \\
\hline $\begin{array}{l}\text { Optimal } \\
\text { cutoff score }\end{array}$ & $\mathrm{N} / \mathrm{A}$ & Ntotal $\geq 3$ & Ptotal $\geq 3$ \\
\hline Sensitivity & N/A & $0.93(0.66-1.00)$ & $0.86(0.57-0.98)$ \\
\hline Specificity & N/A & $0.74(0.66-0.82)$ & $0.75(0.66-0.83)$ \\
\hline PPV & N/A & $0.30(0.17-0.46)$ & $0.29(0.16-0.46)$ \\
\hline NPV & N/A & $0.99(0.94-1.00)$ & $0.98(0.92-1.00)$ \\
\hline
\end{tabular}

Note: Figures in parentheses indicate $95 \% \mathrm{Cl}$.

Abbreviations: AUC, area under the curve; $\mathrm{Cl}$, confidence interval; ESSENCE-O, ESSENCE-Questionnaire; ESSENCE-Q-M, ESSENCE-Q completed by mother; ESSENCE-Q-N, ESSENCE-Q completed by public health nurse; ESSENCE-Q-P, ESSENCE-Q completed by specialized psychologist; NPV, negative predictive value; $\mathrm{N} / \mathrm{A}$, not applicable; PPV, positive predictive value. 


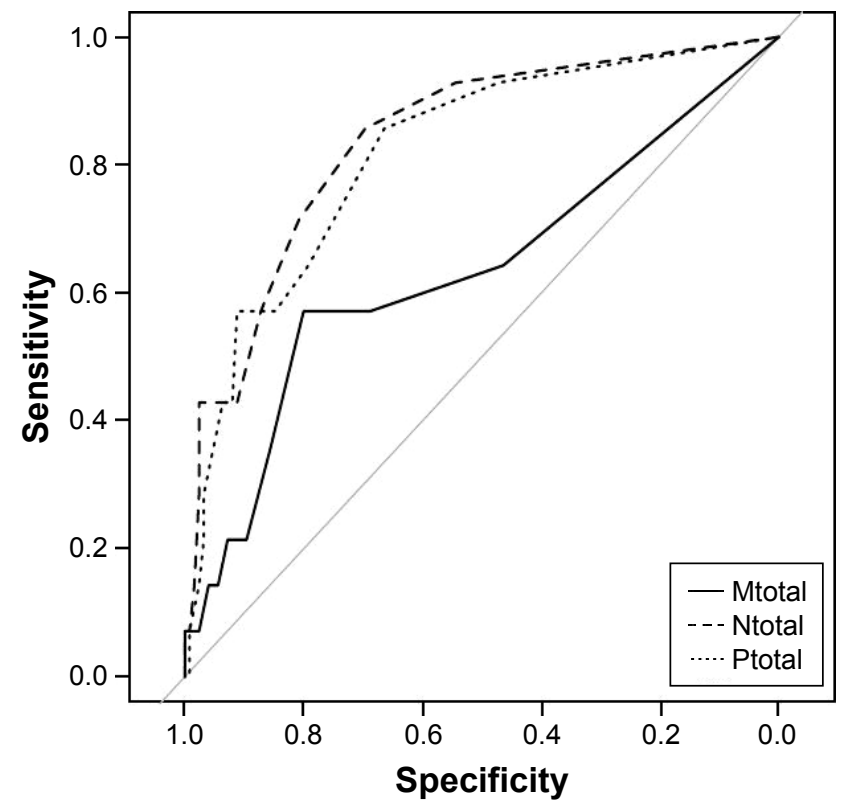

Figure 4 ROC curves for predicting ESSENCE diagnoses at 36-month checkups based on overall ESSENCE-Q score in ESSENCE-Q-M (Mtotal), ESSENCE-Q-N (Ntotal), and ESSENCE-Q-P (Ptotal).

Abbreviations: ESSENCE, early symptomatic syndromes eliciting neurodevelopmental clinical examinations; ESSENCE-Q, ESSENCE-Questionnaire; ESSENCE-Q-M, ESSENCE-Q completed by mother; ESSENCE-Q-N, ESSENCE-Q completed by public health nurse; ESSENCE-Q-P, ESSENCE-Q completed by specialized psychologist; ROC, receiver operating characteristic.

\section{Discussion}

According to the results obtained from these 18- and 36/42-month checkups - with AUCs for ESSENCE-Q-N and ESSENCE-Q-P of 0.8-0.9 and NPVs of almost 1.0 - the ESSENCE-Q used by PHNs and specialized psychologists seems to hold promise as a general population screening tool for neurodevelopmental disorders in young children. However, the validity of ESSENCE-Q-M (showing an AUC of 0.69 in the 18 -month and 0.63 in the 36-month checkup)

Table 4 AUC, sensitivity, specificity, PPV, and NPV of the three ESSENCE-Qs in 36-month checkups

\begin{tabular}{llll}
\hline $\begin{array}{l}36 \text { months } \\
(\mathbf{n}=\mathbf{1 3 9})\end{array}$ & ESSENCE-Q-M & ESSENCE-Q-N & ESSENCE-Q-P \\
\hline AUC & $0.63(0.46-0.8 \mathrm{I})$ & $0.84(0.73-0.95)$ & $0.82(0.69-0.94)$ \\
$\begin{array}{l}\text { Optimal } \\
\text { cutoff score }\end{array}$ & $\mathrm{N} / \mathrm{A}$ & Ntotal $\geq 2$ & Ptotal $\geq 2$ \\
Sensitivity & $\mathrm{N} / \mathrm{A}$ & $0.86(0.57-0.98)$ & $0.86(0.57-0.98)$ \\
Specificity & $\mathrm{N} / \mathrm{A}$ & $0.70(0.61-0.76)$ & $0.66(0.57-0.75)$ \\
PPV & N/A & $0.24(0.13-0.38)$ & $0.22(0.12-0.36)$ \\
NPV & N/A & $0.98(0.92-1.00)$ & $0.98(0.92-1.00)$ \\
\hline
\end{tabular}

Note: Figures in parentheses indicate $95 \% \mathrm{Cl}$.

Abbreviations: AUC, area under the curve; Cl, confidence interval; ESSENCE-Q, ESSENCE-Questionnaire; ESSENCE-Q-M, ESSENCE-Q completed by mother; ESSENCE-Q-N, ESSENCE-Q completed by public health nurse; ESSENCE-Q-P, ESSENCE-Q completed by specialized psychologist; NPV, negative predictive value; N/A, not applicable; PPV, positive predictive value. seems not sufficient for a screening tool when used alone. Given that individuals who dropped out were much more often screen positive than were those who remained in the study (and hence much more likely to be real cases), the estimates of psychometric validity presented here are probably conservative. An underlying reason for the better validity of the ESSENCE-Q rating by PHNs and specialized psychologists, compared to the mothers, might be that the professionals' experiences contributed to a more realistic assessment of the children's development. The ESSENCE-Q in its present format appears to possibly have a better validity for screening boys - at least at the 36/42-monthcheckup - as compared with girls with neurodevelopmental disorders in the general population, but numbers after subdivision according to gender were too small to make other than very tentative suggestions. However, the possibility that symptoms of a developmental disorder in some girls may not be obvious enough at the time of screening was not excluded.

Nurses working in maternal and child health are the key professionals for early identification of neurodevelopmental disorders in many western countries, ${ }^{26}$ and, in Japan, PHNs are expected to take this role. ${ }^{27}$ If a trained PHN's identification of children with ESSENCE at the first stage of screening can be shown to be valid when compared with the result of the ESSENCE-Q assessment by a specialized psychologist, the PHN might then even be suggested to take the role of "specialist" when it comes to referral for full neurodevelopmental assessment. Given that the PHNs who joined this study had enough knowledge and experiences with regard to neurodevelopmental disorders in young children, further studies are needed to determine whether or not the results of this study can be generalized to other, larger general population samples.

In the 18-month checkup, a cutoff value of 3 satisfied our requirement (ie, that both sensitivity and specificity be at least $0.7-0.8$, and sensitivity $>$ specificity) for ESSENCE-Q-N and ESSENCE-Q-P. The high NPVs (0.98 in ESSENCE-Q-N and in ESSENCE-Q-P) suggested that almost all children with score $<3$ in the ESSENCE-Q by a PHN (or by a psychologist) would be free of ESSENCE problems/diagnoses. On the other hand, the low PPVs $(0.30$ in ESSENCE-Q-N and 0.29 in ESSENCE-Q-P) suggested that the result on the ESSENCE-Q should not be taken to mean that all children over the cutoff value would have neurodevelopmental diagnoses. Nevertheless, screenpositive children in this cohort will be followed-up, and if later concerns regarding neurodevelopmental problems arise, they will be referred for new neurodevelopment 
assessment. In the 36/42-month checkup, with a cutoff of 3 , the sensitivity was $0.71-0.64$ and the specificity was 0.81-0.79 for ESSENCE-Q-N and ESSENCE-Q-P. This was slightly under our required level. When the cutoff was set at 2, the sensitivity and the specificity almost satisfied our requirement for ESSENCE-Q-N and ESSENCE-Q-P. NPVs were almost 1.0 and PPVs (0.24 in ESSENCE-Q-N and 0.22 in ESSENCE-Q-P) were similar to levels at 18-month checkups. These cutoff values (3 in 18-month checkups and 2 in 36-month checkups) are almost identical to those suggested by the originator of the ESSENCE-Q ("Yes" $\geq 1$ or "Maybe/a little" $\geq 3$ ).

The results of the ESSENCE-Q by mothers, PHNs, and specialized psychologists were known to the pediatrician who made the decision to refer or not for secondary checkup. This is a clear limitation of the present study. However, given that the study was performed in a routine clinical environment that already included some kind of explicit screening for neurodevelopmental disorders, it would have been ethically problematic to recruit a representative population if parentreported or observed problems would not have influenced the decision to refer for in-depth assessment. The strength of the study includes its population-based nature, the very thorough clinical examination of the children, and the fact that the screening procedure used is clinically applicable as it stands ("quick and easy" and based on a one-page questionnaire).

There are several studies that have claimed that the developmental concerns expressed by parents should be taken seriously by specialists. ${ }^{28,29}$ It was previously shown that the ESSENCE-Q completed by parents of children referred for neurodevelopmental assessment can be a useful screening tool for neurodevelopmental disorders in a clinical setting. ${ }^{11}$ However, given that the numbers were small in both the present and the clinical study, further research is needed before generalizable conclusions can be drawn.

\section{Disclosure}

The authors report no conflicts of interest in this work.

\section{References}

1. Wilson JMG, Junger G. The principles and practice of screening for disease. In: WHO, ed. Vol PA/66.7. Geneva: World Health Organization; 1968.

2. Radecki L, Sand-Loud N, O'Connor KG, Sharp S, Olson LM. Trends in the use of standardized tools for developmental screening in early childhood: 2002-2009. Pediatrics. 2011;128(1):14-19.

3. Berry AD, Garzon DL, Mack P, Kanwischer KZ, Beck DG. Implementing an early childhood developmental screening and surveillance program in primary care settings: lessons learned from a project in Illinois. J Pediatr Health Care. 2014;28(6):516-525.
4. Ozonoff S. Editorial: Early detection of mental health and neurodevelopmental disorders: the ethical challenges of a field in its infancy. J Child Psychol Psychiatry. 2015;56(9):933-935.

5. Glascoe FP. Screening for developmental and behavioral problems. Ment Retard Dev Disabil Res Rev. 2005;11(3):173-179.

6. Fernell E, Hedvall A, Westerlund J, et al. Early intervention in 208 Swedish preschoolers with autism spectrum disorder. A prospective naturalistic study. Res Dev Disabil. 2011;32(6):2092-2101.

7. Blanche EI, Chang MC, Gutierrez J, Gunter JS. Effectiveness of a sensory-enriched early intervention group program for children with developmental disabilities. Am J Occup Ther. 2016;70(5): 7005220010p1-7005220010p8.

8. Geoffray MM, Thevenet M, Georgieff N. News in early intervention in autism. Psychiatr Danub. 2016;28(Suppl 1):66-70.

9. Gillberg C. The ESSENCE in child psychiatry: early symptomatic syndromes eliciting neurodevelopmental clinical examinations. Res Dev Disabil. 2010;31(6):1543-1551.

10. Gillberg C. ESSENCE-Q (Questionnaire); 2012. Available from: http://gillbergcentre.gu.se/english/research/screening-questionnaires/ essence-q. Accessed November 1, 2016.

11. Hatakenaka Y, Fernell E, Sakaguchi M, Ninomiya H, Fukunaga I, Gillberg C. ESSENCE-Q - a first clinical validation study of a new screening questionnaire for young children with suspected neurodevelopmental problems in south Japan. Neuropsychiatr Dis Treat. 2016; 12:1739-1746.

12. Miniscalco C, Nygren G, Hagberg B, Kadesjö B, Gillberg C. Neuropsychiatric and neurodevelopmental outcome at 6 and 7 years of children who screened positive for language problems at 30 months. Dev Med Child Neurol. 2006;48:361-366.

13. Araga N, Gokan Y. Maternal and child health law. In: Araga N, Goan Y, editors. Public Health Nursing in Japan. Tokyo: Intermedical; 2011:9.

14. Community Health \& Health Promotion Project Report; 2014. Available from: http://www.mhlw.go.jp/toukei/saikin/hw/c-hoken/14/d1/kekka1. pdf. Accessed November 1, 2016.

15. Hatakenaka Y, Hirano S. Training health professionals engaging in 18-month checkup for early detection and early intervention of autism spectrum disorder. J Soc Policy Soc Work. 2015;19:45-57.

16. Charman T, Baird G. Practitioner review: diagnosis of autism spectrum disorder in 2- and 3-year-old children. J Child Psychol Psychiatry. 2002;43(3):289-305.

17. Charman T, Taylor E, Drew A, Cockerill H, Brown JA, Baird G. Outcome at 7 years of children diagnosed with autism at age 2: predictive validity of assessments conducted at 2 and 3 years of age and pattern of symptom change over time. $J$ Child Psychol Psychiatry. 2005; 46(5):500-513.

18. Corsello CM, Akshoomoff N, Stahmer AC. Diagnosis of autism spectrum disorders in 2-year-olds: a study of community practice. $J$ Child Psychol Psychiatry. 2013;54(2):178-185.

19. Koyama T, Osada H, Tsujii H, Kurita H. Utility of the Kyoto Scale of Psychological Development in cognitive assessment of children with pervasive developmental disorders. Psychiatry Clin Neurosci. 2009; 63(2):241-243.

20. Nakai K, Suzuki K, Oka T, et al. The Tohoku Study of Child Development: a cohort study of effects of perinatal exposures to methylmercury and environmentally persistent organic pollutants on neurobehavioral development in Japanese children. Tohoku J Exp Med. 2004;202(3):227-237.

21. Wing L, Leekam SR, Libby SJ, Gould J, Larcombe M. The diagnostic interview for social and communication disorders: background, interrater reliability and clinical use. J Child Psychol Psychiatry. 2002; 43(3):307-325.

22. Goodman R. The strengths and difficulties questionnaire: a research note. J Child Psychol Psychiatry. 1997;38(5):581-586.

23. Battaglia A, Carey JC. Diagnostic evaluation of developmental delay/ mental retardation: an overview. Am J Med Genet C Semin Med Genet. 2003;117C(1):3-14. 
24. Mackrides PS, Ryherd SJ. Screening for developmental delay. Am Fam Physician. 2011;84(5):544-549.

25. Worobey J. Developmental testing. In: Hopkins B, editors. The Cambridge Encyclopedia of Child Development. Cambridge: Cambridge University Press; 2005:114-117.

26. Barbaro J, Ridgway L, Dissanayake C. Developmental surveillance of infants and toddlers by maternal and child health nurses in an Australian community-based setting: promoting the early identification of autism spectrum disorders. J Pediatr Nurs. 2011;26(4):334-347.

27. Ishii M, Matsuda N, Takeda S. Challenges faced by Japanese public health nurses in supporting children with autism spectrum disorder and their families: a qualitative study. B Health Sci Kobe. 2013; 29:37-44.
28. Sacrey LA, Zwaigenbaum L, Bryson S, et al. Can parents' concerns predict autism spectrum disorder? A prospective study of high-risk siblings from 6 to 36 months of age. J Am Acad Child Adolesc Psychiatry. 2015;54(6):470-478.

29. Richards M, Mossey J, Robins DL. Parents' concerns as they relate to their child's development and later diagnosis of autism spectrum disorder. J Dev Behav Pediatr. 2016;37:532-540.

\section{Publish your work in this journal}

Neuropsychiatric Disease and Treatment is an international, peerreviewed journal of clinical therapeutics and pharmacology focusing on concise rapid reporting of clinical or pre-clinical studies on a range of neuropsychiatric and neurological disorders. This journal is indexed on PubMed Central, the 'PsycINFO' database and CAS, and is the official journal of The International Neuropsychiatric Association (INA). The manuscript management system is completely online and includes a very quick and fair peer-review system, which is all easy to use. Visit http://www.dovepress.com/testimonials.php to read real quotes from published authors.

Submit your manuscript here: http://www.dovepress.com/neuropsychiatric-disease-and-treatment-journal 